\title{
Evaluating the Incidence Rate of an Accelerated Short Course High Dose Rate Intravaginal Brachytherapy Complications in Patients with Endometrial Cancer
}

\author{
Farnaz Amouzegar-Hashemi ${ }^{1}$, Zakieh Vesgari Kiasari ${ }^{1}$, Bita Kalaghchi ${ }^{1}$, Mahdi \\ Aghili ${ }^{1}$, Soraya Gholami ${ }^{2}$, Sepideh Mansouri ${ }^{1,3}$, Sepand Moalej $^{4}$, Afsaneh Maddah \\ Safaei $^{1 *}$
}

\begin{abstract}
Background: Brachytherapy in treatment of endometrial cancer patients is growing and therefore, evaluation of more feasible schedule has become of great importance. The purpose of current study was to evaluate the complications of accelerated short course high dose rate intravaginal brachytherapy (HDR IVB), a new brachytherapy approach which is a more feasible treatment option in developing countries. Method: From 2017 to 2018, 54 patients diagnosed with endometrial cancer and FIGO stages IA to IIB who underwent total abdominal hysterectomy with a bilateral salpingo-oophorectomy were enrolled in present study. They were treated with a total dose of $25 \mathrm{~Gy}$ in 5 fractions which was prescribed daily. A dose of $5 \mathrm{~Gy}$ was prescribed at a depth of $0.5 \mathrm{~cm}$ in the upper third and middle third of vagina. Adverse effects related to organs at risk consist of bladder, vagina and rectum were documented based on the Common Terminology Criteria for Adverse Events v3.0 (CTCAE v3.0). Results: The accelerated short course HDR IVB was well tolerated and no grade 3 or higher toxicities was reported for patients during the follow up period. There were no chronic rectal toxicities and only one patient showed chronic urinary toxicities. However, the incidence rate of vaginal toxicities at the end of 4-month and 8-month follow up periods was higher than acute toxicities and significantly lower in elderly group compared to younger group. Conclusion: Overall, the accelerated HDR IVB was safe and was well tolerated in endometrial cancer patients and the incidence rate of undue complications were equal, if not less, in elderly patients compared to the younger ones.
\end{abstract}

Keywords: Accelerated short course high dose rate brachytherapy- complications- endometrial cancer- organ at risk

Asian Pac J Cancer Prev, 20 (7), 2039-2043

\section{Introduction}

Cancer of the endometrium is the most frequently diagnosed gynecologic malignancy in united states and the fifth most prevalent cancer in Iran (Yazdani et al., 2017). Endometrium carcinoma is staged surgically based on the criteria established by the International Federation of Gynecology and Obstetrics (FIGO) with most of the cases presenting an early stage of the disease. Primary approaches toward management of endometrial cancer consists of total hysterectomy and bilateral salpingo-oophorectomy with or without pelvic and paraaortic lymph node dissection (Creasman et al., 1987; Creutzberg et al., 2000; Keys et al., 2004). However, locoregional relapse may occur in $9-14 \%$ of patients with adenocarcinoma of the endometrium Stages I and II following surgery alone (Creutzberg et al., 2000; Keys et al., 2004). In addition, elderly patients ( $>60$ y) (Creutzberg et al., 2000) as well as the ones with poor differentiated (Aalders et al., 1980), and/or lymphovascular space involved tumors (Keys et al., 2004) are at a much higher risk of locoregional relapse.

Postoperative pelvic external beam radiation therapy (EBRT) is an effective therapeutic strategy for decreasing the locoregional recurrence rate in patients with Stages I and II endometrial carcinoma (Creutzberg et al., 2000; Keys et al., 2004). However, since $75 \%$ of locoregional failures are isolated vaginal relapses, more restricted forms of adjuvant radiation therapy, including intravaginal high-dose-rate brachytherapy (HDR IVB) have become much more popular. Additionally, HDR IVB is cheaper, safer and requires less department workload compared to pelvic EBRT (Martínez-Monge et al., 2007). Based on the Postoperative Radiation Therapy in Endometrial

${ }^{1}$ Radiation Oncology Research Center (RORC), Cancer Institute, ${ }^{2}$ Physics Department of Radiation Oncology, Tehran University of Medical Sciences, ${ }^{3}$ Recombinant Proteins Department, Breast Cancer Research Center, Motamed Cancer Institute, ACECR, Tehran, Iran, ${ }^{4}$ Western University of Health Sciences, Pomona, CA. *For Correspondence: afsan1980@ymail.com 
Carcinoma (PORTEC)-2 trial, IVB alone was as effective as EBRT in controlling vaginal relapses of patients high intermediate-risk (HIR) endometrial cancer and could effectively minimize gastrointestinal toxicity (Creutzberg et al., 2000).

Based on evidences, there were described 24 schedules for HDR IVB which are different in case of total dose, dose per fraction and duration of treatment. Nevertheless, no standard regimen has been developed till now (Sabater et al., 2017). According to the 2014 American Brachytherapy Society update survey (Harkenrider et al., 2017), the majority of patients received 21 Gy of HDR brachytherapy in three fractions. This long-term duration of therapy makes it inconvenient for patients living far away from the radiation center and declines compliance of the patients to the therapy. To overcome this problem, Gaztañaga and colleagues developed an accelerated IVB schedule of 25 Gy in five consecutive treatment sections, from Monday to Friday. This therapeutic schedule was shown to be bioequivalent in terms of tumor $(\alpha / \beta=10)$ and late $(\alpha / \beta=3)$ effects with the conventional $21 \mathrm{~Gy} / 3$ Rx scheme (Gaztanaga et al., 2012).

The need for brachytherapy and specially this newly developed method is uprising in cancer therapy for the elderly; nevertheless, this subgroup of patients often suffers from concomitant and chronic medical problems including high pressure, cardiovascular disorders and diabetes (Sablińska, 1979). Although few data exist on the effectiveness and tolerability of radiotherapy for aged patients with uterine cervix carcinoma (Sablińska, 1979; Kennedy et al., 1989; Mitsuhashi et al., 1995; Sakurai et al., 2000), none exist regarding the outcome of therapy for accelerated HDR IVB. Therefore, it is an interesting issue to evaluate whether accelerated HDR IVB results in undue complications in elderly patients just like younger ones or not. Furthermore, to our best knowledge, no studies have assessed the feasibility and probable toxicities associated with this method in Iranian population. In present study, we evaluated the complication rates and their intensity for the $25 \mathrm{~Gy} / 5 \mathrm{Rx} / 5$ days accelerated HDR IVB schedule treatment comparing patients aged $\geq 60$ years and $<60$ years with stage I-II endometrial cancer in Iranian subpopulation.

\section{Materials and Methods}

From 2017 to 2018, fifty-four patients diagnosed with endometrial cancer and FIGO Stages IA to IIB who underwent total abdominal hysterectomy with a bilateral salpingo-oophorectomy were enrolled in present study, conducted at radiation oncology department of Imam Khomeini hospital, affiliated to Tehran University of Medical Sciences, Iran, from which 21 were more than 60 years old (assigned as elderly group) and 33 were 60 or younger (assigned as younger group). The eligibility criteria consisted of patients only with endometrioid adenocarcinoma or adenosquamous carcinoma. Patients with atypical histologies including clear-cell carcinoma or papillary serous carcinoma were considered ineligible for current study. Further details of the studied population are presented in Table 1.
The median interval between the operation date and beginning of brachytherapy was 42 days (range 28-112). In the first consultation session, patient's detailed clinical history was recorded and careful physical examinations were performed. The length and diameter of the vaginal remnant were recorded. If the vaginal cuff showed signs of incomplete healing after surgery, the brachytherapy treatment was postponed for a median of 1-2 weeks.

In general, the principles of the American Brachytherapy Society (Nag et al., 2000) were followed in the choice of applicators and in dosimetric evaluation. Vaginal cylinders of 2.0 to $3.5 \mathrm{~cm}$ in diameter that comfortably fit into the vagina were used in all cases. Colpostats were not used. A dose of 5 Gy was prescribed at a depth of $0.5 \mathrm{~cm}$ in the upper third and middle third of the lateral wall of the vagina and at a depth of $0.3 \mathrm{~cm}$ in the middle third. The lower third of the vagina was not treated. Different dose constraint points were placed on the surface of the upper third of the vagina as well as on the surface and at 0.5 $\mathrm{cm}$ depth at the vaginal apex. Bladder and rectal doses were not routinely calculated. Patients were scheduled to receive five treatments of intravaginal HDR brachytherapy over 5 consecutive days, although in some cases, the treatments were protracted due to departmental logistics. The median duration of the HDR brachytherapy treatments was 5 days (range 5-12).

Once the HDR brachytherapy was completed, all patients were evaluated for bladder, rectal, and vaginal toxicities immediately after therapy and at first-month, fourth-month and eight-month follow up sessions. Adverse effects were documented based on the Common Terminology Criteria for Adverse Events v3.0 (CTCAE v3.0). Toxicities observed during the first 90-day period from completion of therapy were defined as "acute toxicities", while the ones occurring after this period were classified as "late toxicities".

Statistical analyses were performed administrating frequency tables and to figure out the associations between variables, Chi-square and Fisher exact tests were used.

\section{Results}

The incidence rate of acute and chronic rectal, vaginal and urinary side effects of HDR brachytherapy in both groups has been presented in Table 2. Overall, the intervention was well-tolerated by patients in studied groups and no grade 3 nor higher toxicities were reported for all studied patients during the follow up period.

The incidence rate of acute rectal side effects was low and no significant differences were reported both immediately after and 1-month post brachytherapy. Only one patient in elderly group was reported with a grade 2 rectal toxicity immediately after intervention. Similar patterns of toxicity were also observed for acute vaginal and urinary related toxicities. No grade 2 urinary toxicities were observed in studied population. In conclusion, the studied acute phase toxicities were mild and insignificant between studied groups.

Regarding chronic rectal side effects, no toxicities were observed neither after 4-month nor 8-month follow up periods and no significant differences were observed 
Table 1. Demographic Data of Patients

\begin{tabular}{lccc}
\hline Variable & $\begin{array}{c}\text { Elderly patients } \\
(>60)(\mathrm{N}=21)\end{array}$ & $\begin{array}{c}\text { Younger group } \\
(\leq 60)(\mathrm{N}=33)\end{array}$ & $\begin{array}{c}\mathrm{P} \\
\text { value }\end{array}$ \\
\hline Histologic Grade & & & \\
1 & $18(78.3 \%)$ & $28(84.8 \%)$ & 0.74 \\
$2-3$ & $3(21.7 \%)$ & $5(15.2 \%)$ & \\
LVI & & & \\
Present & $3(21.7 \%)$ & $5(15.2 \%)$ & 0.68 \\
Absent & $18(78.3 \%)$ & $28(84.8 \%)$ & \\
LND & & & \\
Performed & $13(61.9 \%)$ & $16(48.5 \%)$ & 0.33 \\
Not performed & $8(38.1 \%)$ & $17(51.5 \%)$ & \\
Stage & & & \\
1 & $4(19.0 \%)$ & $8(24.3 \%)$ & 0.93 \\
2 & $17(81.0 \%)$ & $25(75.7 \%)$ & \\
\hline
\end{tabular}

between groups. In addition, only one patient showed urinary chronic toxicity. Contrarily, chronic vaginal side effects demonstrate an uprising incidence trend over the follow up period. Interestingly, the incidence rate of chronic vaginal side effects was significantly lower in elderly group, compared to the younger one which may propose that younger patients are more prone to vaginal related side effects. The intensity of acute and chronic side effects was almost similar among the studied groups. Regardless of the increasing pattern of chronic vaginal toxicities during the follow up period, other side effects were mild and tolerable.

\section{Discussion}

Endometrial carcinoma is considered as one of the most prevalent female genitourinal neoplasms, and usually with favorable prognosis if a proper radical surgery is carried out on time. Nevertheless, as the low state of women's health due to the presence of concurrent disorders including hypertension, diabetes and obesity is the main cause of endometrial carcinoma arousal, radical surgery is not always feasible in all cases. In these situations, radiotherapy is the mainstay in management and control of the disease (Montemaggi and Guerrieri, 2001).

Age is considered as a significant prognostic value in endometrial carcinoma since a direct correlation exists between aging and involvement of myometrium, an important histologic feature of the disease (Javert, 1958; Sall et al., 1970; Jones, 1975). In a radical radiotherapy treatment, brachytherapy is always considered as a relevant section of therapy. Therefore, studying the tolerability and complications of brachytherapy in elderly population is of utmost importance.

One of the great benefits of brachytherapy is its ability of delivering large doses of radiation specifically to the tumor site while sparing the neighboring normal tissues. As a result, brachytherapy has broadly been applied for decades, either as an approach for delivering an extra dose after a primary course of external beam radiotherapy or as an exclusive treatment modality. The other advantage
Table 2. Incidence Rate of Acute and Chronic Toxicities Related to Accelerated HDR IVB

\begin{tabular}{lccc}
\hline Variable & $\begin{array}{c}\text { Elderly patients } \\
(>60)(\mathrm{N}=21)\end{array}$ & $\begin{array}{c}\text { Younger group } \\
(\leq 60)(\mathrm{N}=33)\end{array}$ & P value \\
\hline Vaginal (acute) & & & \\
Immediately after & $4(19 \%)$ & $2(6.0 \%)$ & 0.25 \\
1-month & $2(9.5 \%)$ & $2(6.0 \%)$ & 0.63 \\
Vaginal (chronic) & & & \\
4-month & $2(9.5 \%)$ & $8(24.0 \%)$ & 0.37 \\
8-month & $2(9.5 \%)$ & $11(33.3 \%)$ & $0.04 \%$ \\
Rectal (acute) & & & \\
Immediately after & $2(9.5 \%)$ & $1(3.0 \%)$ & 0.42 \\
1-month & $0(0.0 \%)$ & $0(0.0 \%)$ & 1 \\
Rectal (chronic) & & & 1 \\
4-month & $0(0.0 \%)$ & $0(0.0 \%)$ & 1 \\
8-month & $0(0.0 \%)$ & $0(0.0 \%)$ & 1 \\
Urinary (acute) & & & 1 \\
Immediately after & $7(33.3 \%)$ & $6(19.3 \%)$ & 0.2 \\
1-month & $3(14.3 \%)$ & $2(6.0 \%)$ & 0.36 \\
Urinary (chronic) & & & $1(3.0 \%)$ \\
4-month & $1(4.7 \%)$ & $0(0.0 \%)$ & 1 \\
8-month & $0(0.0 \%)$ & & \\
\hline & & & \\
\hline
\end{tabular}

of brachytherapy stems from the little duration of time required for delivery of the dose. This is most beneficial in countries where few radiotherapy devices or facilities are available since it is together with numerous sequential socio-economical advantages (Montemaggi and Guerrieri, 2001).

Both low- and high-dose-rate brachytherapies have been successfully applied in treatment of elderly patients with no significant differences in overall outcomes compared to those of the younger ones, possessing similar clinical presentations (Perez and Brady, 1999). In present study, we administered a feasible schedule which prescribed 25 Gy in 5 fractions which were provided more accessible treatment for patients living far from radiation centers. In addition, we evaluated the acute and chronic complications of an accelerated HDR IBT in Iranian population and analyzed the side effects and tolerability of this schedule and also compared the complications in an elderly group with younger group with similar clinical presentations. Although the accelerated form of current therapy might have resulted in occurrence of increased long-term complications, no grade 3 or higher toxicities were observed in patients after the median follow up of 14 months. Our results were consistent with the one reported by Martínez-Monge et al., (2007) and Rios et al., (2016). Similar to previous evidences, accelerated schedules were tolerable with acceptable outcomes (Sabater et al., 2017). Despite this, it must also be mentioned that the lack of high grade chronic side effects may also be due to the low follow up period. Therefore, studies monitoring patients for much longer periods are strongly recommended for further confirmation of the current study's results.

Several treatment parameters including the total dose, fractionation schedule, dose rate and the length of the vagina have shown to affect the toxicities observed with 
vaginal brachytherapy. For instance, the study performed by Sorbe and Smeds (1990), treating patients with 9.0 $\mathrm{Gy} \times 4,6.0 \mathrm{~Gy} \times 5,5 \mathrm{~Gy} \times 6$ and $4.5 \mathrm{~Gy} \times 6$ all prescribed to the $1.0 \mathrm{~cm}$ depth from the edge of the vagina, revealed that increasing the dose of each fraction enhanced observed rectal and bladder related toxicities and significantly increased vaginal atrophy as well as vaginal shortening. Furthermore, they reported that increasing the length of vagina is correlated with increased probability of toxicity occurrence. In addition, the study by Rovirosa et al., (2017) which was investigated three different schedules of vaginal cuff brachytherapy in early stage endometrial cancer, showed that the most prevalent complication is vaginal disorders. Comparing the vaginal control and complications between three schedules including 4-6 Gy $\times 6,5-6$ Gy $\times 4$ and 6 Gy $\times 3$ revealed that 6 Gy for 3 daily fractions is the best treatment option. Similarly, another study by Rovirosa et al., (2015) demonstrated that there were no differences in chronic complications of rectum, bladder and vagina between two schedules including 4-6 Gy $\times 6$ and 5-6 Gy $\times 4$.

One of the findings of our study was that the incidence rate of chronic vaginal side effects was significantly lower in aged patients compared to the younger ones. As the volume of vagina is normally smaller in elderly patients, this may in part explain why we observed less chronic vaginal toxicities in elderly studied group. Other studies have also reported a positive correlation between an increase dose of each fraction or the length of vagina with the incidence rate of side effects (Nori et al., 1994; Bahng et al., 2012). The study by Rovirosa et al., (2018) tried to show the correlation between age groups and vaginal cuff relapse, cancer specific survival and late toxicities of rectum, bladder and vagina. It revealed that although patients older than 85 years has worse outcome, there were not any differences between age groups and rectal, bladder and vaginal late toxicities. Although statistically not significant, a study comparing the complications of high dose rate vaginal brachytherapy (HDR VBT) with low dose rate vaginal brachytherapy (LDR VBT) demonstrated more side effect occurrence with HDR VBT (Fayed et al., 2007). In theory, utilization of ovoid applications may also be beneficial in lowering toxicities by more restricting the dose of the apex of vagina and pushing away the bladder as well as rectum from the site of therapy. Unfortunately, there are no currently existing data which compares the effects of the applicators shape on the incidence rate of the complication (Harkenrider et al., 2015).

Overall, the accelerated HDR IVB was safe and well-tolerated in all patients and it can be a more feasible schedule in developing country where patients have limited access to radiation wards. Regardless of chronic vaginal toxicities, the incidence rate and intensity of other undue complications, either acute or chronic, were equal between elderly patients and the younger ones. Finally, the patients in younger group were more prone to chronic vaginal complications compared to the elderly ones.

\section{References}

Aalders J, Abeler V, Kolstad P, et al (1980). Postoperative external irradiation and prognostic parameters in stage I endometrial carcinoma: clinical and histopathologic study of 540 patients. Obstet Gynecol, 56, 419-27.

Bahng AY, Dagan A, Bruner DW, et al (2012). Determination of prognostic factors for vaginal mucosal toxicity associated with intravaginal high-dose rate brachytherapy in patients with endometrial cancer. Int J Radiat Oncol Biol Physics, 82, 667-73.

Creasman WT, Morrow CP, Bundy BN, et al (1987). Surgical pathologic spread patterns of endometrial cancer: a Gynecologic Oncology Group study. Cancer, 60, 2035-41.

Creutzberg CL, van Putten WL, Koper PC, et al (2000). Surgery and postoperative radiotherapy versus surgery alone for patients with stage-1 endometrial carcinoma: multicentre randomised trial. Lancet, 355, 1404-11.

Fayed A, Mutch DG, Rader JS, et al (2007). Comparison of high-dose-rate and low-dose-rate brachytherapy in the treatment of endometrial carcinoma. Int J Radiat Oncol Biol Phys, 67, 480-4.

Gaztanaga M, Cambeiro M, Villafranca E, et al (2012). Long-term results of 1-week intravaginal high-dose-rate brachytherapy alone for endometrial cancer. Brachytherapy, 11, 119-24.

Harkenrider MM, Block AM, Alektiar KM, et al (2017). American Brachytherapy Task Group Report: Adjuvant vaginal brachytherapy for early-stage endometrial cancer: A comprehensive review. Brachytherapy, 16, 95-108.

Harkenrider MM, Block AM, Siddiqui ZA, et al (2015). The role of vaginal cuff brachytherapy in endometrial cancer. Gynecol Oncol, 136, 365-72.

Javert CT (1958). Prognosis of endometrial cancer. Obstet Gynecol, 12, 556-71.

Jones HW (1975). Treatment of adenocarcinoma of the endometrium. Obstet Gynecol Surv, 30, 147-69.

Kennedy AW, Flagg JS, Webster KD (1989). Gynecologic cancer in the very elderly. Gynecol Oncol, 32, 49-54.

Keys HM, Roberts JA, Brunetto VL, et al (2004). A phase III trial of surgery with or without adjunctive external pelvic radiation therapy in intermediate risk endometrial adenocarcinoma: a Gynecologic Oncology Group study. Gynecol Oncol, 92, 744-51.

Martínez-Monge R, Nagore G, Cambeiro M, et al (2007). Intravaginal 1-week high-dose-rate brachytherapy alone for Stages I-II endometrial cancer. Brachytherapy, 6, 195-200.

Mitsuhashi N, Takahashi M, Nozaki M, et al (1995). Squamous cell carcinoma of the uterine cervix: radiation therapy for patients aged 70 years and older. Radiology, 194, 141-5.

Montemaggi P, Guerrieri P (2001). Brachytherapy in the elderly. Crit Rev Oncol Hematol, 37, 159-67.

Nag S, Erickson B, Parikh S, et al (2000). The American Brachytherapy Society recommendations for high-dose-rate brachytherapy for carcinoma of the endometrium. Int $J$ Radiat Oncol Biol Phys, 48, 779-90.

Nori D, Merimsky O, Batata M, et al (1994). Postoperative high dose-rate intravaginal brachytherapy combined with external irradiation for early stage endometrial cancer: a long-term follow-up. Int J Radiat Oncol Biol Phys, 30, 831-7.

Perez C, Brady L (1999). Principles and practice of radiation oncology. J Pediatr Hematol Oncol, 21, 560.

Rios I, Rovirosa A, Ascaso C, et al (2016). Vaginal-cuff control and toxicity results of a daily HDR brachytherapy schedule in endometrial cancer patients. Clin Transl Oncol, 18, 925-30.

Rovirosa A, Ascaso C, Arenas M, et al (2015). Can we shorten 
the overall treatment time in postoperative brachytherapy of endometrial carcinoma? Comparison of two brachytherapy schedules. Radiother Oncol, 116, 143-8.

Rovirosa A, Cortes KS, Ascaso C, et al (2018). Are endometrial cancer radiotherapy results age related?. Clin Transl Oncol, 20, 1416-21.

Rovirosa A, Herreros A, Camacho C, et al (2017). Comparative results of three short brachytherapy schedules as exclusive treatment in postoperative endometrial carcinoma. Brachytherapy, 16, 1169-74.

Sabater S, Andres I, Lopez-Honrubia V, et al (2017). Vaginal cuff brachytherapy in endometrial cancer - a technically easy treatment?. Cancer Manag Res, 9, 351-62.

Sablińska B (1979). Carcinoma of the uterine cervix in women over 70 years of age. Gynecol Oncol, 7, 128-35.

Sakurai H, Mitsuhashi N, Takahashi M, et al (2000). Radiation therapy for elderly patient with squamous cell carcinoma of the uterine cervix. Gynecol Oncol, 77, 116-20.

Sall S, Sonnenblick B, Stone ML (1970). Factors affecting survival of patients with endometrial adenocarcinoma. $\mathrm{Am}$ J Obstet Gynecol, 107, 116-23.

Sorbe BG, Smeds A-C (1990). Postoperative vaginal irradiation with high dose rate afterloading technique in endometrial carcinoma stage I. Int J Radiat Oncol Biol Phys, 18, 305-14.

Yazdani S, Bouzari Z, Naeimirad M (2017). Epidemiology of Endometrial Cancer in North of Iran. Basic Clin Cancer Res, 8, 37-41.

\section{๑อง}

This work is licensed under a Creative Commons AttributionNon Commercial 4.0 International License. 